\title{
The properties of bright globular clusters, ultra-compact dwarfs and dwarf nuclei in the Virgo core: hints on origin of ultra-compact dwarf galaxies (UCDs)
}

Chengze Liu ${ }^{1}$, Eric W. Peng ${ }^{2}$, Patrick Côté ${ }^{3}$, Hong-Xin Zhang ${ }^{4}$, Laura Ferrarese ${ }^{3}$, Andrés Jordán ${ }^{5}$, J. Christopher Mihos ${ }^{6}$, Roberto P. Muñoz ${ }^{5}$, Thomas H. Puzia ${ }^{5}$, Ariane Lançon ${ }^{7}$, Stephen Gwyn ${ }^{3}$, Jean-Charles Cuillandre ${ }^{8}$, John P. Blakeslee ${ }^{3}$, Alessandro Boselli ${ }^{9}$, Patrick R. Durrell ${ }^{10}$, Pierre-Alain Duc ${ }^{7}$, Puragra Guhathakurta ${ }^{11}$, Lauren A. MacArthur ${ }^{12}$, Simona Mei ${ }^{13,14,15}$, Rubén Sánchez-Janssen ${ }^{16}$ and Haiguang $\mathrm{Xu}^{1}$

${ }^{1}$ Department of Astronomy, Shanghai Jiao Tong University, Shanghai 200240, China, email: czliu@sjtu.edu.cn

${ }^{2}$ Department of Astronomy, Peking University, Beijing 100871, China

${ }^{3}$ NRC Herzberg Astronomy and Astrophysics Research Centre, 5071 West Saanich Road, Victoria, BC V9E 2E7, Canada

${ }^{4}$ Department of Astronomy, University of Science and Technology of China, Hefei 230026, China

${ }^{5}$ Institute of Astrophysics, Pontificia Universidad Católica de Chile, 7820436 Macul, Santiago, Chile

${ }^{6}$ Department of Astronomy, Case Western Reserve University, Cleveland, OH, USA

${ }^{7}$ Observatoire Astronomique de Strasbourg, Université de Strasbourg, CNRS, UMR 7550, 11 rue de l'Université, F-67000 Strasbourg, France

${ }^{8}$ AIM Paris Saclay, CNRS/INSU, CEA/Irfu, Université Paris Diderot, Orme des Merisiers, F-91191 Gif-sur-Yvette Cedex, France

${ }^{9}$ Aix-Marseille Université, CNRS, LAM (Laboratoire d'Astrophysique de Marseille) UMR 7326, 13388, Marseille, France

${ }^{10}$ Department of Physics and Astronomy, Youngstown State University, One University Plaza, Youngstown, OH 44555, USA

${ }^{11} \mathrm{UCO} /$ Lick Observatory, Department of Astronomy and Astrophysics, University of California Santa Cruz, 1156 High Street, Santa Cruz, CA 95064, USA

${ }^{12}$ Department of Astrophysical Sciences, Princeton University, Princeton, NJ 08544, USA

${ }^{13}$ GEPI, Observatoire de Paris, CNRS, University of Paris Diderot, Paris Sciences et Lettres (PSL), 61, Avenue de l'Observatoire 75014, Paris France

${ }^{14}$ University of Paris Denis Diderot, University of Paris Sorbonne Cité (PSC), 75205 Paris Cedex 13, France

${ }^{15}$ California Institute of Technology, Pasadena, CA 91125, USA

${ }^{16}$ UK Astronomy Technology Centre, Royal Observatory Edinburgh, Blackford Hill, Edinburgh, EH9 3HJ, UK

\begin{abstract}
Based on the data from the Next Generation Virgo cluster Survey (NGVS), we statistically study the photometric properties of globular clusters (GCs), ultra-compact dwarfs (UCDs) and dwarf nuclei in the Virgo core (M87) region. We found an obvious negative color $(g-z)$ gradient in GC system associate with M87, i.e. GCs in the outer regions are bluer. However, such color gradient does not exist in UCD system, neither in dwarf nuclei systemaround M87.
\end{abstract}


In addition, we found that many UCDs are surrounded by extended, low surface brightness envelopes. The dwarf nuclei and UCDs show different spatial distributions from GCs, with dwarf nuclei and UCDs (especially for the UCDs with visible envelopes) lying at larger distances to the Virgo center. These results support the view that UCDs (at least for a fraction of UCDs) are more tied to dwarf nuclei than to GCs.

Keywords. galaxies: dwarf; galaxies: nuclei; galaxies: star clusters: general; globular clusters: general

\section{Introduction}

Since the discovery of the ultra-compact dwarf galaxies (UCDs) around 2000 (Hilker et al. 1999, Drinkwater et al. 2000), it has not been clear exactly what they are. There are two main formation scenarios have been proposed: (1) UCDs are just larger, more massive, otherwise normal GCs (e.g., Mieske et al. 2002, Fellhauer \& Kroupa 2002); (2) UCDs are the remnant nuclei of stripped nucleated dwarf galaxies (e.g., Bekki et al. 2001, Binggeli et al. 1985). In the past few years, people found many pieces of evidence which support the tidal stripping scenario (e.g., Pfeffer et al. 2013, Seth et al. 2014, Liu et al. 2015a, Liu et al. 2015b, Mihos et al. 2015, Zhang et al. 2015, Voggel et al. 2016, Ahn et al. 2017, Afanasiev et al. 2018, Ahn et al. 2018, Schweizer et al. 2018, Zhang et al. 2018). Thanks to the high quality images from Next Generation Virgo cluster Survey (NGVS, see Ferrarese et al. 2012), we have homogeneous sample of bright GCs, UCDs and dwarf nuclei simultaneously. In this contribution, we will directly compare the properties of these three type of objects in Virgo core (M87) region and give hints on UCD origin.

\section{Data and sample}

The data we used is drawn from NGVS, which is a deep multi-wavebands $\left(u^{*}, g, r, i\right.$ and $z$ ) imaging survey (see Ferrarese et al. 2012), and NGVS-IR, which is a near-infrared ( $J$ and $K_{s}$ ) imaging survey (see Muñoz et al. 2014). Both these two surveys were carried out with CFHT. In this contribution, we only use the data in Virgo core region.

Bright GC sample. The GC selection is mainly based on color-color diagrams and concentration parameters (see Peng et al. 2018, in preparation). To compare the properties with UCDs, we only use bright GCs in the magnitude range $18.5<g<21.5$ mag. We have a total of $548 \mathrm{GC}$ candidates in the bright GC sub-sample.

UCD sample. The UCD candidates are selected using a combination of several criteria including magnitude $(18.5<g<21.5 \mathrm{mag})$, half-light radius $\left(11<r_{h}<100 \mathrm{pc}\right)$, color-color diagram $\left(u-i\right.$ vs. $\left.i-K_{s}\right)$, surface brightness, and visual inspection. We found 92 UCD candidates associated with M87 (see Liu et al. 2015b).

Dwarf nuclei. The nucleated dwarf ellipticals (dE,Ns) are from either Binggeli et al. $19 \overline{85 \text { or Ferrarese }}$ et al. (2018, in press). There are 38 dwarf nuclei in the magnitude range $18.5<g<21.5 \mathrm{mag}$ in M87 region. We compare the properties of nuclei of these dE,Ns (not the whole galaxy) with GCs and UCDs.

\section{Results}

Color distribution. It is well known that GC systems in massive elliptical galaxies exhibit bimodal color distribution (e.g. Peng et al. 2006). As we can see in right panels of figure 1, not only the bright GCs, but also the UCDs and nuclei show bimidal color $(g-z)$ distribution. As in Liu et al. (2015b), we divide UCDs, bright GCs and nuclei 


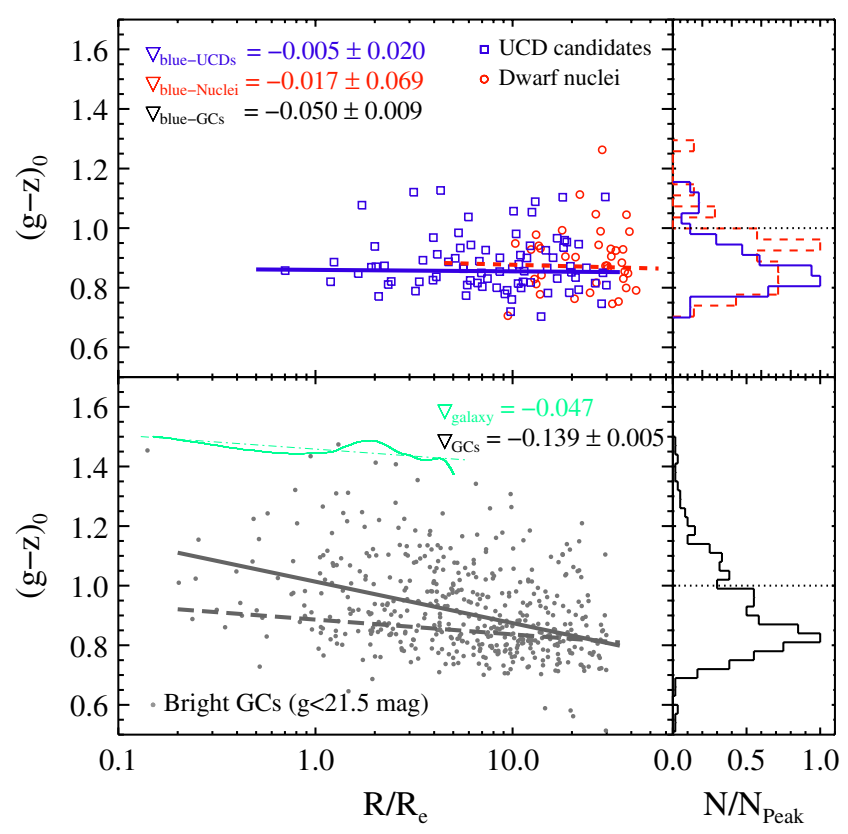

Figure 1. Color distributions (right panels) and color gradients (left panels) for UCDs (blue histogram and blue squares), dwarf nuclei (red histogram and red circles) and bright GCs (black histogram and dark gray dots). We show the color profile for M87 itself (green lines) as well.

into blue and red sub-populations at $(g-z)=1.0$. The fractions of blue sub-populations for three type of objects are $f_{\text {blue-GCs }}=73.3 \%, f_{\text {blue-UCDs }}=89.0 \%, f_{\text {blue-Nuclei }}=89.5 \%$ respectively. The blue fraction of UCDs and nuclei are approximately equal, while the blue GCs fraction is 15 percentage points smaller.

Color gradients. Since most of the UCDs and nuclei are blue, we only measure and compare the color gradients for the blue sub-populations. The corresponding color gradients are $\nabla_{\text {blue-GCs }}=-0.050 \pm 0.009, \nabla_{\text {blue }- \text { UCDs }}=-0.005 \pm 0.020$ and $\nabla_{\text {blue }- \text { Nuclei }}=$ $-0.017 \pm 0.069$. Again, GCs show different properties from UCDs and nuclei. The blue GC system show an obvious negative gradient $(\sim 5 \sigma)$ which is consistent with previous studies (e.g., Liu et al. 2011). But neither blue UCD system nor blue nuclei system exhibit significant color gradients. We notice that the M87 itself has an obvious negative color gradients as well (see green lines in figure 1). The most straightforward interpretation of the color gradient is that GCs were formed together with the host galaxy but UCDs and $\mathrm{dE}$,Ns were formed in a different way.

Spatial distribution. We found that many UCDs are embedded in extended, low surface brightness envelopes (see images in figure 2), which should be the feature of dE,Ns. We divide UCDs into two sub-samples according to whether they have visible envelopes or not. Now we have four types of objects: GCs, UCDs, UCDs with envelopes and dwarf nuclei. The lower-left panel of figure 2 shows the cumulative distribution of project distances to M87 for these four types of objects. The mean distance for dwarf nuclei is the largest, followed by UCDs with visible envelope, UCD without visible envelope and GCs. Actually, this is also in order of the size of envelope. It seems that the gravitational potential well governs the envelope structure of UCDs and dwarf nuclei. This result supports the tidal stripping scenario. 


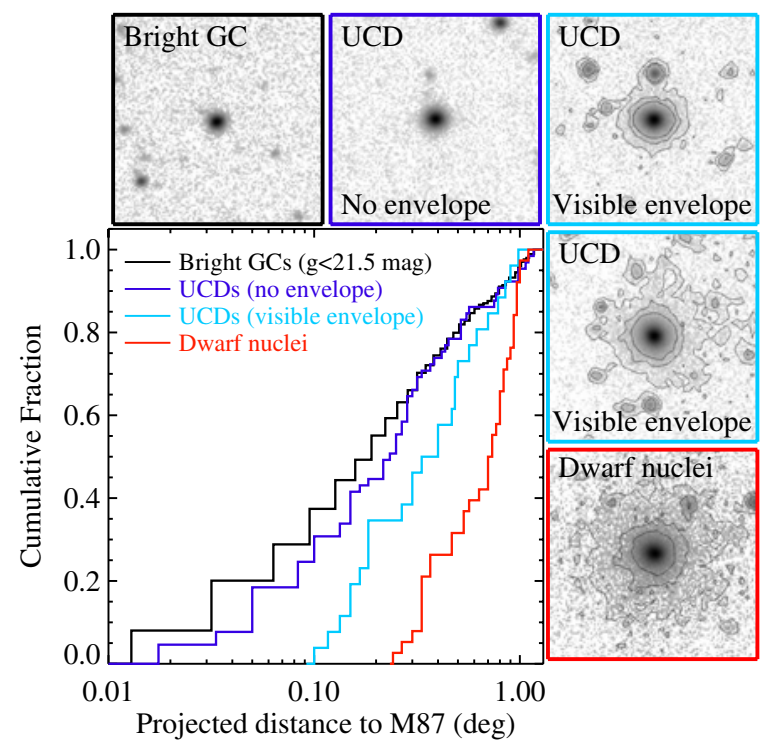

Figure 2. Cumulative distribution of project distances to M87 for bright GCs (black line), UCDs without visible envelope (blue line), UCDs with visible envelopes (cyan line) and dwarf nuclei (red line). The 5 small panels show the selected $g$ band images of 1 bright GC, 3 UCDs and 1 dwarf nucleus. The contours in the right three panels denotes the isophote with constant surface brightness level: 25.0, 25.5, $26 \mathrm{mag} \operatorname{arcsec}^{-2}$ respectively.

\section{Conclusion}

We compare the color distributions, the color gradients and the spatial distributions for bright GCs, UCDs and dwarf nuclei. Statistically, the UCDs show similar properties to dwarf nuclei, and GCs are the outlier. Our results support the tidal stripping scenario. However, we did not rule out any other scenario. It is entirely possible that there are multiple formation pathways.

\section{Acknowledgement}

The authors acknowledge the NSFC grants 11673017, 11833005, 11621303, 11203017, 11433002. C.L. is supported by Key Laboratory for Particle Physics, Astrophysics and Cosmology, Ministry of Education.

\section{References}

Afanasiev, A. V., Chilingarian, I. V., Mieske, S., et al. 2018, MNRAS, 477, 4856

Ahn, C. P., Seth, A. C., den Brok, M., et al. 2017, ApJ, 839, 72

Ahn, C. P., Seth, A. C., Cappellari, M., et al. 2018, ApJ, 858, 102

Bekki, K., Couch, W. J., \& Drinkwater, M. J. 2001, ApJ (Letters), 552, L105

Drinkwater, M. J., Gregg, M. D., Hilker, M., et al. 2003, Nature, 423, 519

Drinkwater, M. J., Jones, J. B., Gregg, M. D., \& Phillipps, S. 2000, PASA, 17, 227

Fellhauer, M., \& Kroupa, P. 2002, MNRAS, 330, 642

Ferrarese, L., Côté, P., Cuillandre, J.-C., et al. 2012, ApJS, 200, 4

Hilker, M., Infante, L., Vieira, G., Kissler-Patig, M., \& Richtler, T. 1999, A\&ASS, 134, 75

Liu, C., Peng, E. W., Jordán, A., et al. 2011, ApJ, 728, 116

Liu, C., Peng, E. W., Toloba, E., et al. 2015a, ApJ (Letters), 812, L2

Liu, C., Peng, E. W., Côté, P., et al. 2015b, ApJ, 812, 34

Mieske, S., Hilker, M., \& Infante, L. 2002, A\&GA, 383, 823

Mihos, J. C., Durrell, P. R., Ferrarese, L., et al. 2015, ApJ (Letters), 809, L21 
Muñoz, R. P., Puzia, T. H., Lançon, A., et al. 2014, ApJS, 210, 4

Peng, E. W., Jordán, A., Côté, P., et al. 2006, ApJ, 639, 95

Schweizer, F., Seitzer, P., Whitmore, B. C., Kelson, D. D., \& Villanueva, E. V. 2018, ApJ, 853,54

Seth, A. C., van den Bosch, R., Mieske, S., et al. 2014, Nature, 513, 398

Voggel, K., Hilker, M., \& Richtler, T. 2016, A\& A, 586, A102

Zhang, H.-X., Peng, E. W., Côté, P., et al. 2015, ApJ, 802, 30

Zhang, H.-X., Puzia, T. H., Peng, E. W., et al. 2018, ApJ, 858, 37 